\title{
The Role of Environmental Management Performance in Higher Education Institutions
}

\author{
Nicolas Roos ${ }^{1, *}$, Xaver Heinicke ${ }^{2, *}$, Edeltraud Guenther ${ }^{3}$ and Thomas W. Guenther ${ }^{2}$ (D) \\ 1 Sustainability Management and Environmental Accounting, Faculty of Business Management and \\ Economics, Technische Universitaet Dresden, 01069 Dresden, Germany \\ 2 Management Accounting and Control, Faculty of Business Management and Economics, Technische \\ Universitaet Dresden, 01069 Dresden, Germany; thomas.guenther@tu-dresden.de \\ 3 United Nations University Institute for Integrated Management of Material Fluxes and of Resources \\ Dresden, 01067 Dresden, Germany; guenther@unu.edu \\ * Correspondence: nicolas.roos@tu-dresden.de or nicolas.roos@tu-drwesden.de (N.R.); \\ xaver.heinicke@tu-dresden.de (X.H.); Tel.: +49-351-4633-3822 (N.R.)
}

Received: 28 November 2019; Accepted: 11 January 2020; Published: 16 January 2020

\begin{abstract}
Higher education institutions (HEIs) are influential social institutions which educate future decision-makers and shape society as a whole. Motivated by new public management, the proliferation of business tools, and a rising awareness for responsible acting, environmental management has also become a matter for HEIs. Focusing on performance outcomes and improvement based mechanisms leads to a professionalization through the active management of environmental issues. Therefore, the support of management structures is an essential prerequisite when implementing environmental efforts. Thus far, little attention has been dedicated to environmental management performance and steering processes of environmental issues in HEIs, which marks the research gap of this study. This article presents results of a survey on the concept of environmental management performance (EMP) based on Trumpp et al. (2015) aiming to answer the research question of how HEIs conduct environmental management along the dimensions of EMP, which includes environmental policy, environmental objectives, environmental processes, organizational structures, and monitoring. The results show that, as of now, HEIs pursue no common practice when approaching EMP. Nevertheless, two thirds of the respondents show an orientation towards sustainability with particularly high values regarding issues of environmental policy.
\end{abstract}

Keywords: higher education institutions; sustainability assessment; environmental management performance; German-speaking countries; survey

\section{Introduction}

Higher education institutions (HEIs), as important societal pillars, play a key role in shaping future generations and paving the way for sustainable development [1,2]. In their role as educational institutions, the creation of knowledge and the promotion of ideas for society are their major undertakings. Besides the core tasks of teaching and research, environmental efforts concerning the institutions' operational performance have gradually emerged. With the conservation of resources, saving energy, or the reduction of waste [3], an integrated understanding of taking responsibility for their own actions has spread within many organizations, including HEIs.

With increased awareness of one's own social responsibility, the wish to fulfill it and to set a good example also rises [1]. As a result, practices dedicated to the systematic management of these tasks, such as environmental management systems, have been established and promoted.

Despite these instruments originally being designed for the business context, they quickly became useful in public organizations such as HEIs. Over time, these became increasingly common as a tool for 
managing environmental responsibility on campuses of various European countries [4]. The associated thinking of improvement-based mechanisms and performance enhancement instigates a general professionalization of organizational processes, including the handling of organizational responsibility with regard to sustainable action [1]. This occurred in the context of a general transfer of business practices to public administration, resulting in a more holistic approach and professionalization of the organizations [4].

In order to meet these growing challenges, and to meet the requirements of a whole-institution approach, the role of management is becoming increasingly important for the professionalization and control of organizational activities.

Management plays a core role in the allocation of resources, strategic alignment, and the planning, implementation, and evaluation of activities [5,6]. This gives HEIs a greater significance in dealing with ecological sustainability aspects by extending their responsibilities from a purely operational level to a more strategic one. Despite expanding to include management functions, the performance of HEIs regarding ecological aspects is largely excluded from management considerations. Concepts that examine environmental management performance (EMP) in the context of HE remain nonexistent thus far.

As the systematic literature review by Roos and Guenther [7] shows, an examination of EMP has so far played a minimal role in the assessment of (ecological) sustainability at HEIs. Although individual aspects of environmental management at HEIs can be assigned to the dimensions of the EMP according to Trumpp et al. [8], no systematic approach based on performance standards is pursued. For this purpose, a step-by-step integration of control mechanisms within the framework of a holistic institutional approach is suggested, which is oriented towards a growing integration of management capacities at HEIs. However, most of the research to date has been devoted to case studies of other topics, such as the investigation of success factors and obstacles or the implementation of sustainability in HEIs [1,8-10], while others examined the assessment of sustainability engagement [3,11,12]. While this research strand mainly draws on case studies, another research strand, which is represented in more recent research, uses surveys to investigate new insights into implementation efforts to generate sustainability. This research direction is increasingly turning to systematic approaches that examine the management of sustainability and environmental aspects at the management level. Although these approaches are aimed at managing sustainability at an executive level, this research is based on general practice-driven implementation efforts with the aim of initiating engagement. However, approaches examining environmental sustainability from a systematic management performance-driven perspective have not yet been investigated [13-15]. This represents a blind spot in the research on controlling sustainability at the management level and thus marks the research gap in the present work.

On the basis of these insights, the present survey study provides first evidence on the management of environmental sustainability in HEIs along the dimensions of EMP according to Trumpp et al. [8]. Trumpp et al. distinguish between environmental management performance (EMP) and environmental operational performance (EOP). We focused our survey on EMP. The associated research question is therefore dedicated to the following topic:

How do HEIs conduct environmental management along the dimensions of EMP, including environmental policy, environmental objectives, environmental processes, organizational structures, and monitoring?

In our view, this shows a major weakness in the efforts to adopt a whole-institution approach. Although the measurement of EMP is an important aspect, without an overall institutional view of the interrelationships in the network of effects with policies, objectives, structures and processes, their long-term significance and effects remain limited. A task will be to anticipate the growing need for forward-looking management and to perceive EMP as an overall institutional task. Thus, it is a management mission to address and combine the necessary tasks of sustainability management. 
To answer this research question, the study investigates the present environmental sustainability efforts of HEIs to assess the application of environmental management. Therefore, the survey applied the dimensions of EMP [8] to identify relevant management structures and dimensions. Though the origin of the EMP construct is from the business context, the concept is also applicable for other organizations, such as HEIs [8].

The cross-sectional survey study addressed all public HEIs in German-speaking countries (i.e., Germany, Austria, and Switzerland).

Our study makes two contributions to the literature. First, survey-based research provides the opportunity to investigate relatively complex, multi-faceted phenomena which occur in their natural setting, and surveys are particularly suitable for gathering data on respondents' beliefs, attitudes, and perceptions that drive their behavior [16]. Thus, the survey method allows for mapping current practices in the field, providing insights into intriguing research topics that require further research or that have not yet been studied [16]. The survey study adds to the mostly qualitative research in this area $[17,18]$ reporting on implementation approaches, value-driven behaviors, or discussing factors of success by exploring how HEIs incorporate environmental concerns in their management bodies.

Second, the study expands the existing literature on sustainability in public HEIs by investigating the managerial perspective of engagement. Since current studies on sustainability at HEIs mainly discuss possible pathways of implementation or the assessment of existing practices, the survey contributes to a holistic perspective by examining the management process along the different dimensions of EMP [16]. The study highlights the role of management functions, which enables new perspectives on the often-ignored aspects of systematical internal steering and processing by the top management. This produces a better understanding of objectives and conditions of the successful implementation of sustainability as a cross-cutting function within HEIs.

The remainder of this paper is structured as follows. In the next section, we describe the survey design and the measurement of the constructs. In Section 3, we present the descriptive results. Finally, we discuss our findings and present implications, suggestions for future research, and limitations of our study.

\section{Materials and Methods}

\subsection{Data Collection and Sample}

The population comprises all public HEIs in Germany, Austria, and Switzerland with a total number of 339. The HE systems of these three countries are comparable in their composition with a strong state regulation in the HE sector [19]. A standardized questionnaire was developed based on the recommendations of Dillman, Smyth and Christian [20] and pretested with five experienced sustainability practitioners and five experts from the research field. The experts from the HE sustainability practice had recommended a subdivision into "measures" (e.g., energy efficiency) and "fields" of action (e.g., teaching and research). The survey was developed in English to adopt items that had been published in English and afterwards translated into German. The wording of selected items was adjusted to the HE context. In order to ensure consistency in the two languages, an independent translator was asked to back-translate the survey [21]. After revising the questionnaire, we sent it to the person responsible for environmental issues in the individual HEIs in December 2017. We have proceeded, as follows, to select the appropriate respondent. We first tried to contact the person responsible for sustainability. If this was not possible, we searched for a person responsible for environmental issues, then for a person responsible for occupational health and safety or, finally, for a person responsible for public relations with a request to forward the questionnaire to a person responsible for sustainability and environmental issues.

The survey implementation contained the following four steps: (i) initial e-mailing, (ii) first follow-up, (iii) second follow-up, and (iv) third follow-up. The first step involved an e-mail that was sent to every contact person, including a cover letter and the questionnaire as an interactive PDF 
form. The first follow-up was a replacement of the questionnaire and contained the same elements as described in the first step but was sent to those who had not answered, while the second and third follow-ups were phone calls.

In total, the survey generated a response of 33 (GER: 27; AUT: 4, $\mathrm{CH}: 2$ ) usable questionnaires, which were sent exclusively to state HEIs, resulting in a response rate of $9.7 \%$. The response rate is hardly comparable with other studies as surveys in HE on the topic of sustainability are scarce. However, the findings show that sample selection bias is not a major concern [22,23], following the suggestions of Armstrong and Overton [24]. First, in Table 1 we first match the HEI's characteristics of our sample with known attributes of the population (i.e., organizational size, country of origin, and type of HEI). HEIs with less than 7000 students are categorized as small, with at least 7000 and less than 24,000 students as medium, and with at least 24,000 students as large (similar to the classification of Burrell et al. [15]). Using a chi-square difference test, a significant difference in responses is only found for type of HEIs, which means that fewer universities of applied science and more universities responded than expected. Second, in Table 2 we compare latent variable scores between early and late respondents to test for non-response bias following the idea that "less readily" available responses (i.e., late respondents) are equivalent to non-responses. We define early respondents as those who answered at least with the first follow-up. No significant differences were found. For this reason, it is rather unlikely that there is a systemic bias on the basis of differences between early and late respondents. Nevertheless, we are aware of the relative low number of total responses as a limitation of our findings. Response rates around $10 \%$ can also be found in other recently published surveys (e.g., Guenther/Heinicke [22] with 11.26\%, Bisbe and Malagueno [25] with 13.21\%; de Geuser et al. [26] with $9.5 \%$; Libby and Lindsay [27] with $13.6 \%$ and $1.5 \%$ response rate for two sub-samples). The decline in response rates is also reported in recent methodological papers (e.g., Hiebl and Richter [28]; van der Stede et al. [29]). Thus, we finally decided not to apply statistical tests in our Results section. For the Results section, we use boxplots to display the data of the survey more on an explorative level.

Table 1. Non-response test for organizational size, type of HEI (Higher Education Institution), and country of origin.

\begin{tabular}{|c|c|c|c|c|c|c|c|c|}
\hline $\begin{array}{l}\text { Organi-zational } \\
\text { Size }\end{array}$ & Received & Expected & Type of HEI & Received & Expected & $\begin{array}{c}\text { Country of } \\
\text { Origin }\end{array}$ & Received & Expected \\
\hline Large & 6 & 3.6 & University & 17 & 10.9 & Germany & 27 & 25.7 \\
\hline Medium & 8 & 8.1 & \multirow{2}{*}{$\begin{array}{c}\text { University of } \\
\text { Applied } \\
\text { Science }\end{array}$} & \multirow{2}{*}{16} & \multirow{2}{*}{22.1} & Austria & 4 & 4.1 \\
\hline Small & 19 & 21.3 & & & & Switzerland & 2 & 3.2 \\
\hline Total & \multicolumn{2}{|c|}{33} & \multicolumn{4}{|c|}{33} & \multicolumn{2}{|c|}{33} \\
\hline $\begin{array}{c}\text { Chi- } \\
\text { square test } \\
\text { statistic }\end{array}$ & \multicolumn{2}{|c|}{1.850} & \multicolumn{4}{|c|}{5.092} & \multicolumn{2}{|c|}{0.459} \\
\hline $\mathrm{df}$ & \multicolumn{2}{|c|}{2} & \multicolumn{4}{|c|}{1} & \multicolumn{2}{|c|}{2} \\
\hline$p$-value & \multicolumn{2}{|c|}{0.397} & \multicolumn{3}{|c|}{$0.024^{* *}$} & & \multicolumn{2}{|c|}{0.796} \\
\hline
\end{tabular}

The Table reports the results of the chi-square statistics for the tests of regional distribution and of type of higher education institution (HEI). ${ }^{*}$ Significant at the 0.1 level. ${ }^{* *}$ Significant at the 0.05 level. ${ }^{* * *}$ Significant at the 0.01 level; two-tailed tests. 
Table 2. Non-response test for latent constructs.

\begin{tabular}{cccc}
\hline Latent Constructs & Early Respondents & Late Respondents & Mann-Whitney U Test \\
\hline Environmental Policy & $4.15(n=22)$ & $3.75(n=11)$ & $Z=-0.860, p=0.390$ \\
Environmental Objectives & $3.76(n=22)$ & $3.45(n=11)$ & $Z=-0.748, p=0.455$ \\
Environmental Processes & $4.22(n=22)$ & $3.33(n=11)$ & $Z=-1.514, p=0.130$ \\
Organizational Structure & $3.82(n=22)$ & $3.55(n=11)$ & $Z=-0.404, p=0.686$ \\
Monitoring Systems & $3.46(n=22)$ & $3.10(n=11)$ & $Z=-0.765, p=0.444$ \\
Overall EMP & $3.88(n=22)$ & $3.42(n=11)$ & $Z=-0.860, p=0.390$ \\
\hline
\end{tabular}

The Table shows the construct means of the early and late respondents of our sample for the latent constructs of the structural model. ${ }^{*}$ Significant at the 0.1 level. ${ }^{* *}$ Significant at the 0.05 level. ${ }^{* * *}$ Significant at the 0.01 level; two-tailed tests.

\subsection{Measures}

By addressing persons in charge of environmental management, we aimed to assess the performance of each institution compared to the average of public HEIs of similar size at the public level. Therefore, we refer to the work of Trumpp et al. [8], who define and conceptualize a measurement of environmental management performance that comprises the five dimensions of environmental policy, environmental objectives, environmental processes, organizational structure, and environmental monitoring to pursue a systematization of engagement in order to map the status quo of environmental management performance.

We use 12 items each to measure environmental policy, environmental objectives, and environmental processes; 13 items to measure environmental monitoring; and 2 items to measure organizational structure. All items are based on a seven-point Likert scale with midpoint and endpoints labeled as categories $(1=$ strongly disagree/not at all; $4=$ neither nor; $7=$ strongly agree/completely).

In close coordination and cooperation (dialogue, meetings and questionnaire pretest) with experts in operational environmental management at the TU Dresden, a total of four core areas of environmental management were identified in addition to the dimensions according to Trumpp et al., which are important for the investigation of EMP in the context of HEIs and were therefore also considered as independent fields in the questionnaire: (1) Energy efficiency, (2) fresh water consumption, (3) resource consumption, and (4) emission reduction. Furthermore, we developed certain domains within HEIs bearing key management responsibilities in terms of environmental performance. These responsibilities spread over the measures of (5) procurement, (6) facility management, (7) event management, (8) disposal, (9) research and education, (10) mobility, (11) human resource management (HRM), and (12) marketing. With this general structure, the questionnaire portrays the essential fields of action and measures to uncover environmental performance within the dimensions of EMP.

\section{Results}

For the visualization of our results, we use SPSS 25.0 and MS Excel software programs to create boxplots. Boxplot diagrams allow for a summarization of a dataset along the values of minimum, lower quartile, median, mean value, upper quartile, and maximum. This kind of illustration enables benchmarking and interpreting the results, especially for smaller sample sizes.

The box describes the lower and upper quartile, with the vertical line crossing it as the median. The whiskers connect the quartiles with the minimum (left) and maximum (right). The position of the triangle displays the mean value (see Figure 1). This shows if performance is below or above average. If a value is located within the light grey area or even above, the performance is between the median and the upper quartile. If a value is located in the dark grey area or even below, the opposite is true. The interquartile distance defines the range of the box, whereas the size of the whole boxplot represents the range of all answers given on the question. 


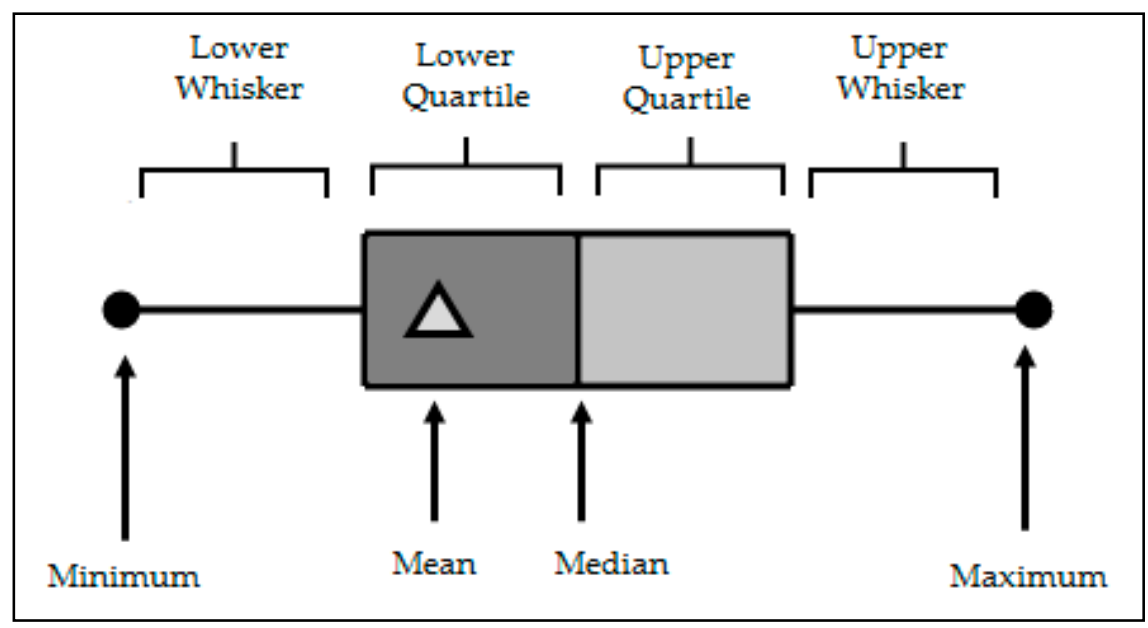

Figure 1. Description of boxplot diagrams (own illustration).

For the analysis, we divided the questionnaire into different sections following the dimensions of EMP. For each question, within the sections, we generated a boxplot diagram. The following section describes the results based on the fields of action and measures proposed above.

The first boxplot addressing environmental policy (see Figure 2) shows that energy efficiency is, on average, the most elaborated field of action (mean $=4.64$ ). Policies concerning the reduction of emissions show a slightly below average level. The examination of water consumption and resource utilization deliver average patterns (mean $=4.15$ and 4.21 , respectively). Turning to HEIs' environmental performance, policy issues concerning waste management show an above mean value (4.79). Considering also the median, disposal policy appears to be a well-elaborated topic. This may be due to legal requirements regulating such matters in German-speaking countries. Concerning mobility, the mean value is 4.21 , which is below the median of 5 , though the average performance is below average.

When it comes to environmental policies for the case of marketing or HRM, the results tend towards a lower level (1st quartile starts with a value of 1 , which means strongly disagree), which is also confirmed by low mean values (3.03 and 3.25, respectively).

For the dimension of environmental objectives (see Figure 3), the measures of energy efficiency (3.94), water consumption (3.58), resource utilization (3.67), and emission reduction (3.48) show a level slightly below average. Consulting the median confirms the trend of a generally only medium adoption in practice. Finally, objectives on energy efficiency show the best average outcome, although the interquartile distance covers a broad range (values between 2 to 6). However, environmental objectives appear to be remarkably well-elaborated concerning disposal (mean $=4.36$ ), as we can see the median reaching a high level (=5.00) compared to the other fields of action. Mobility objectives show a pattern similar to that of the policy dimension, since a mean value of 3.72 lies slightly below the median of 4.00. As was already observable for the dimension of environmental policy, marketing and HRM consistently show a low level of agreement (mean $=3.00$ and 3.03, respectively). This becomes even clearer, since the lower quartile starts with the lowest value of 1 (=strongly disagree). Remarkably, event management shows almost similar values of agreement $($ mean $=3.13)$. 


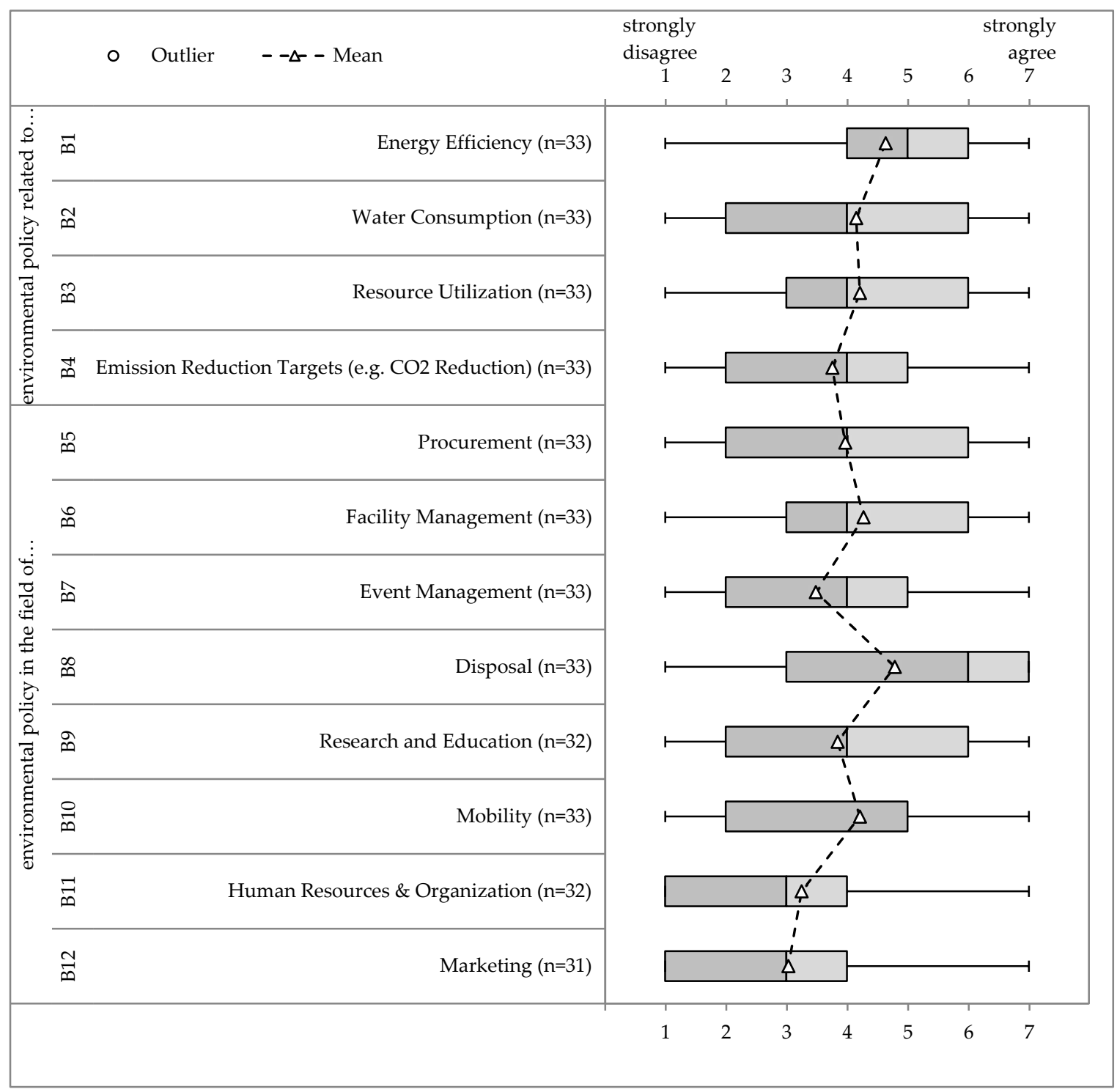

Figure 2. Results for the dimension environmental policy.

From the boxplot for environmental processes (see Figure 4), we can observe the highest approval rates for energy efficiency improvements (mean $=4.27$ ), although only at a slightly better value. Processes improving the consumption of water (3.91), improving resource efficiency (3.85), or reducing emissions (3.79) show an average agreement. Looking at processes concerning environmental performance, they show a similar level of assessment. The mean values are located between 3.34 (event management) and 4.63 (disposal), which provide no considerable indications corresponding with our observations for the dimension of environmental objectives. The higher mean value and median for waste management again results from legal requirements which regulate mandatory waste disposal. The processes of mobility show a mean value of 4.03 corresponding to the median (value $=4.00$ ). In terms of marketing and HRM, the low values (mean for both $=3.26$ ) reveal a weak level of consent. 


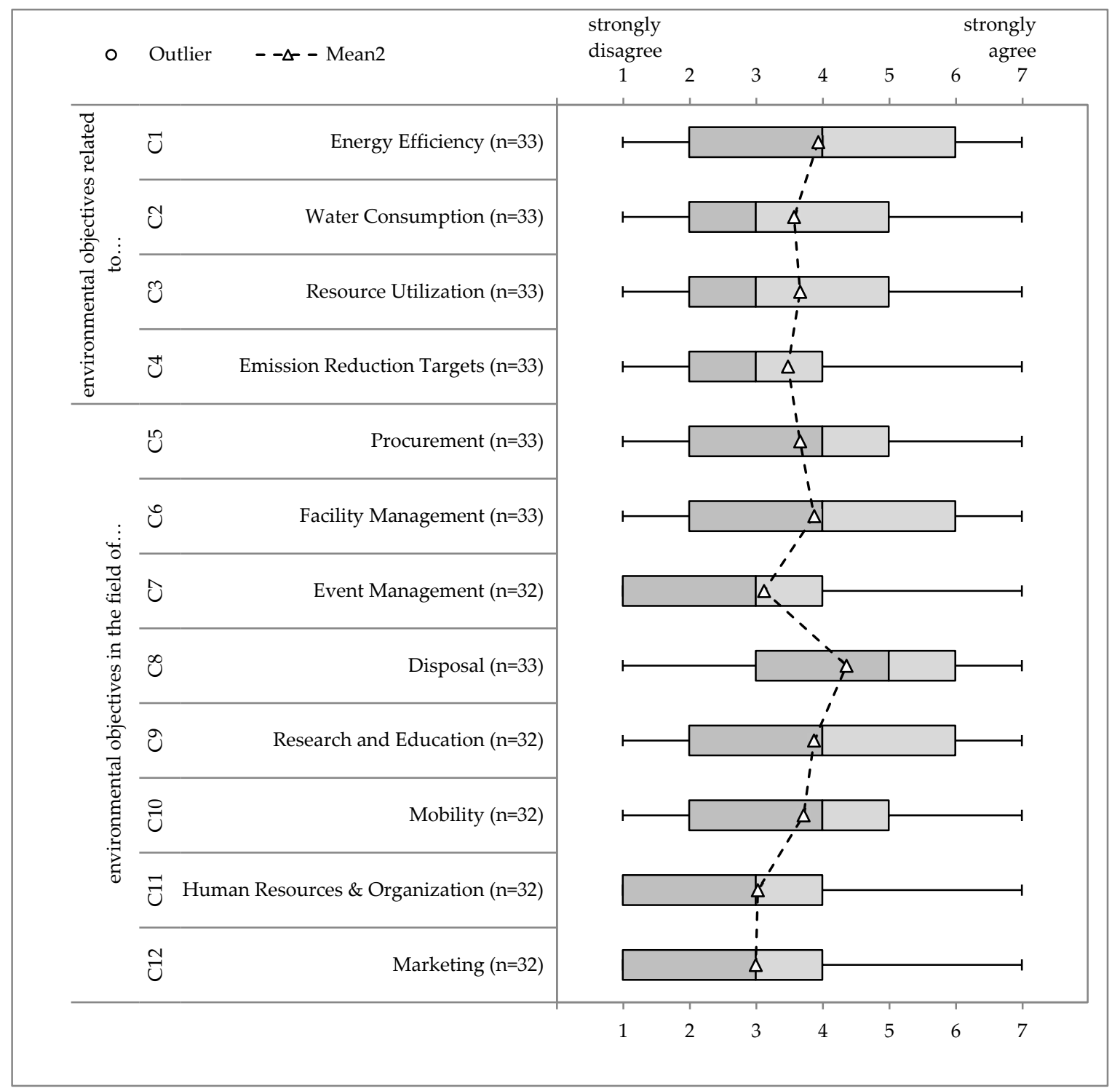

Figure 3. Results for the dimension environmental objectives.

The exploration of the organizational structure (Figure 5) follows an alternative design diverging from the interrogative form of the other dimensions. Since this section asks about implementation efforts of HEIs with the aim of revealing certain structures related to environmental topics, the standardized question pattern is unsuitable for providing appropriate results. Therefore, the dimension for organizational structures captures items on training and internal communication. The results show that trainings for employees on environmental topics are generally perceived below average (3.39). The application of adequate internal communication tools for an improved management of environmental topics centers around a mean value of 4.06. Remarkably, the interquartile distance covers a broad spectrum ranging from 2 (=disagree) to 6 (=agree). 


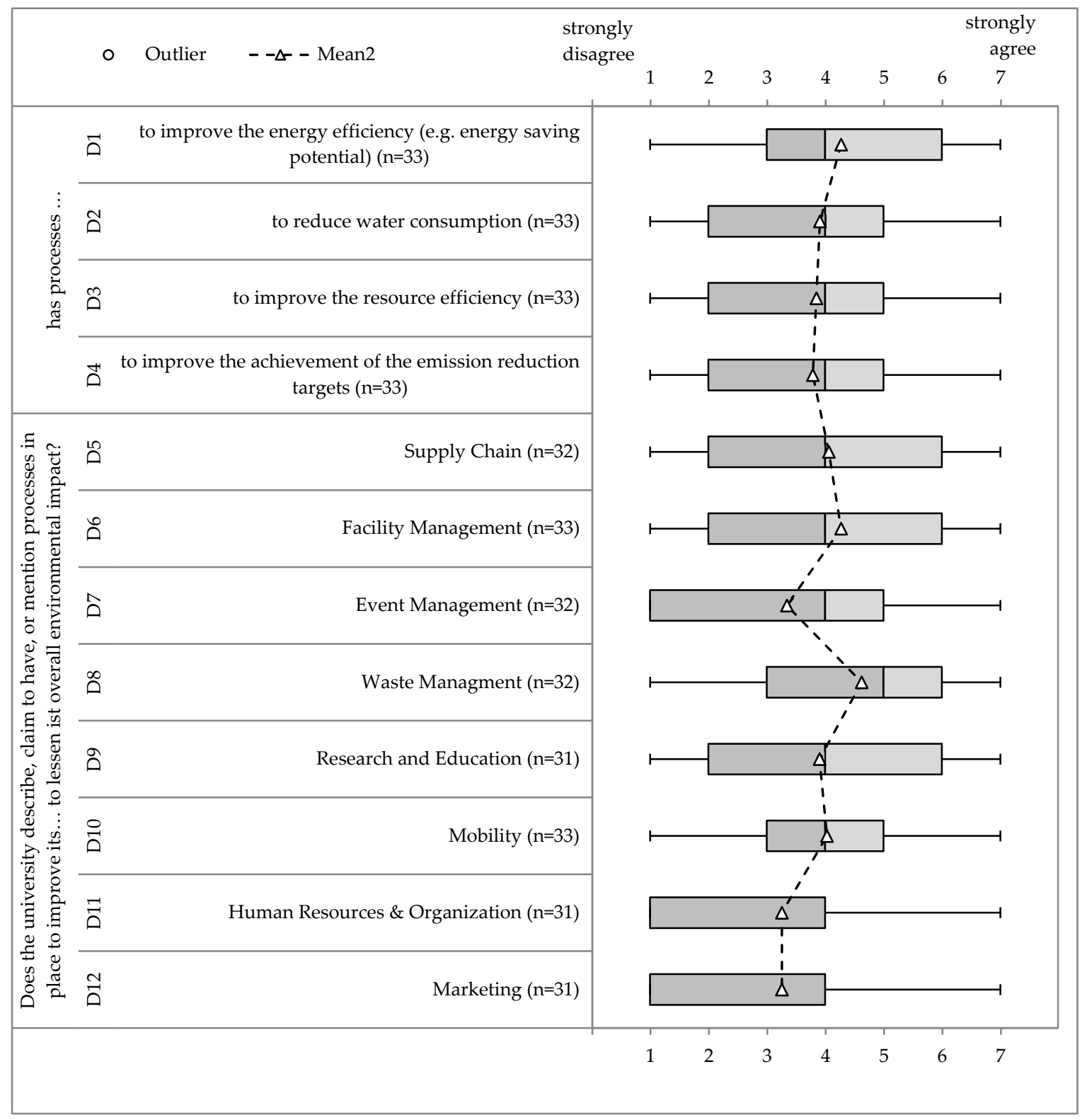

Figure 4. Results for the dimension environmental processes.

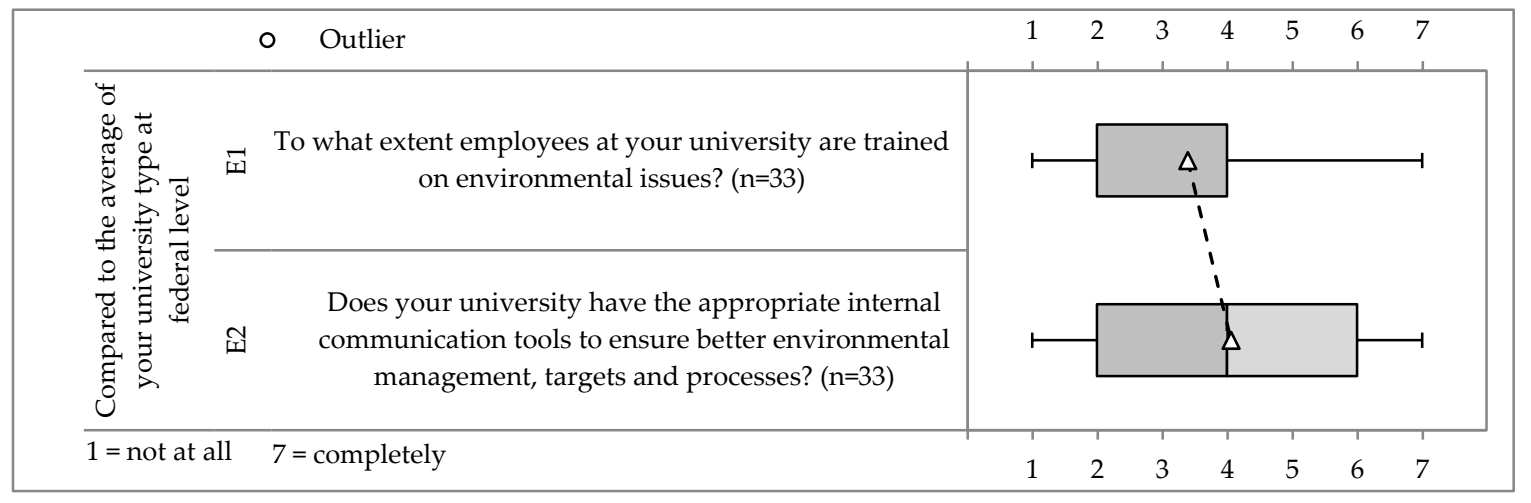

Figure 5. Results for the dimension organizational structure. 
The boxplot in Figure 6, for the dimension of monitoring systems for environmental management performance, indicates that HEIs mainly apply measurement tools for the case of energy efficiency (mean $=4.27$ ). Here we can observe a slightly higher mean value than in other fields of action (water use: 3.67; resource use: 3.24; emission reduction: 3.09). The efficient use of resources, which includes tools for the reduction of emissions and the application of monitoring systems, appears to be slightly underrepresented. In both cases, the lower quartile starts with the value of 1 (=strongly disagree). Regarding the fields of action, HEIs, on average, perform best concerning disposal aspects. With a mean of 4.19 and a median of 5 , again this appears to be the topic with the most emphasis, since regulatory requirements force HEIs to adopt certain disposal procedures. Interestingly, measurement tools for green procurement are obviously not in use, as the mean value of 3.00 indicates a general below average adoption. Similar to the low levels of agreement for the dimensions of policies and objectives, the assessment of monitoring for mobility issues is below average (mean $=3.45$ ), which again is below the median of 4.00. Finally, event management (mean =3.06), marketing (mean $=2.57$ ), and HRM (mean $=2.65$ ) consistently show the same below average performance as the other dimensions. In all these fields, the lower quartile begins with the value of 1 (strongly disagree) and ends with the value of 4 (partly agree). Especially for the case of marketing, the median shows a considerably low level (value $=2.00$ ). The final question on environmental monitoring systems for other relevant fields of HEIs shows a low mean of 2.79 .

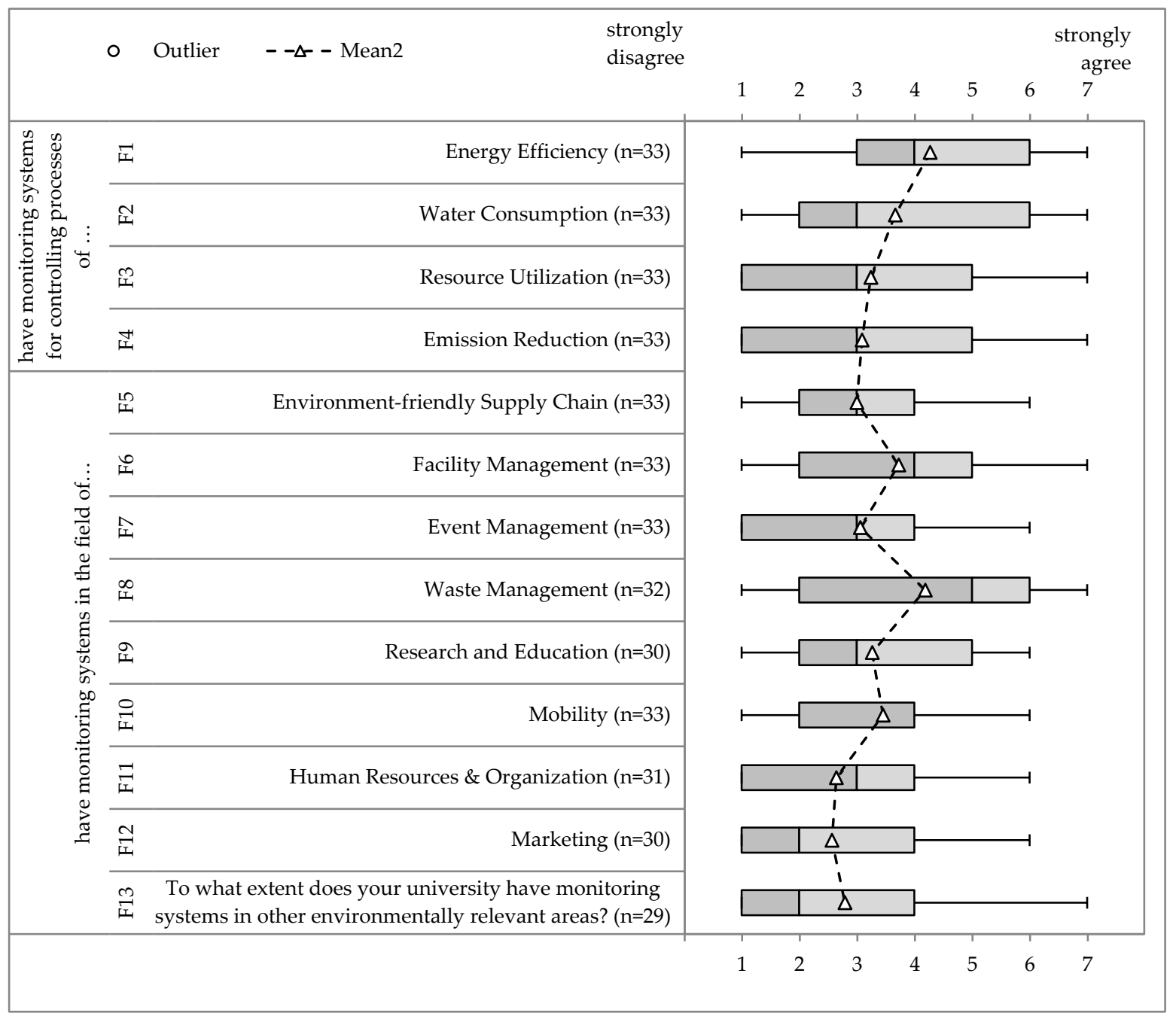

Figure 6. Results for the dimension monitoring systems. 
In summary, the analysis allows deeper insights into the performance dimensions of EMP for HEIs. In the next step, our study examines the mean scores over all items for each EMP dimension (Table 3), which allows for implications on the performance of each single EMP dimension for the HEIs.

Table 3. EMP (Environmental Management Performance) mean scores for the EMP dimensions.

\begin{tabular}{ccccccc}
\hline Score & Dimension of EMP & $\begin{array}{c}\text { Environmental } \\
\text { Policy }\end{array}$ & $\begin{array}{c}\text { Env. } \\
\text { Objectives }\end{array}$ & $\begin{array}{c}\text { Env. } \\
\text { Processes }\end{array}$ & $\begin{array}{c}\text { Org. } \\
\text { Structures }\end{array}$ & $\begin{array}{c}\text { Monitoring } \\
\text { Systems }\end{array}$ \\
\hline Mean Value & 4.0 & 3.6 & 3.9 & 3.7 & 3.3 \\
\hline
\end{tabular}

Apparently, the dimension environmental policy shows the highest mean value of 4.00 . This shows the general commitment of HEIs to proclaim sustainability engagement on an institutional level. A similar value can be found for the dimension environmental processes (mean $=3.9$ ). This supports a certain anchoring of the environmental engagement within the institutions. The organizational structures with a mean of 3.7 are directly interrelated to these processes and objectives (mean $=3.6)$, consequently followed by monitoring systems (mean $=3.3$ ). In sum, the relative moderate commitment for EMP of HEIs may be due to different emphasis of the HEI's top management for environmental issues in general over other issues at the HEI as well as individual commitment within the HEI's top management.

A further split of the results for the different EMP dimensions by size in Table 4 delivers an even more detailed picture of the performance of the HEIs.

Table 4. EMP mean scores and HEI Size.

\begin{tabular}{ccccccc}
\hline Dimension of EMP & $\begin{array}{c}\text { Environmental } \\
\text { Policy }\end{array}$ & $\begin{array}{c}\text { Env. } \\
\text { Objectives }\end{array}$ & $\begin{array}{c}\text { Env. } \\
\text { Processes }\end{array}$ & $\begin{array}{c}\text { Org. } \\
\text { Structures }\end{array}$ & $\begin{array}{c}\text { Monitoring } \\
\text { Systems }\end{array}$ & $\begin{array}{c}\text { Total } \\
\text { EMP }\end{array}$ \\
\hline Small Size & 3.79 & 3.44 & 3.59 & 3.76 & 3.08 & 3.52 \\
Medium Size & 3.90 & 3.43 & 4.20 & 3.69 & 3.69 & 3.78 \\
Large Size & 4.88 & 4.65 & 4.67 & 3.67 & 3.70 & 4.28 \\
\hline
\end{tabular}

Evidently, large institutions perform, on average, better than small or medium ones. Going from large to small, the study observes a slope of mean values in almost every dimension, whereas the gap between large and medium-sized institutions shows to be more considerable than between medium and small ones. Not surprisingly, the mean value of total EMP also reflects these size effects.

Furthermore, when dividing the sample into two groups of high and low performers, we examine what makes the difference between good and bad performance in terms of EMP. For this case, the mean EMP scores for each EMP dimension are taken and aggregated to one total EMP score of each HEI. We classify HEIs as high performers when the mean score value is greater than 5.00 and as low performers for mean score values of less than 3.00. Cases between 3.00 and 5.00 are categorized as medium [30].

In total, the study identified 7 high performers, 11 low performers, and 15 medium performers. Based on this categorization of HEIs, an additional in-depth analysis of each category was conducted.

Publicly available information on the integration of environmental topics in HEIs' policies, as well as objectives, structures, processes or monitoring on the websites of the HEIs, was examined for further information on environmentally relevant performance characteristics in order to enrich the existing data from the survey to find out more about further efforts on (environmental) sustainability within the curriculum, research projects or voluntary self-obligations. Furthermore, the study sought proof of institutional anchoring within management bodies or formal commitments in the published statements on mission, values, or strategy. In addition, this information was matched with a possible assignment within the EMAS register to see if an institution's performance is based on external validation.

For the case of high-performing HEIs, the study identified a continuous orientation towards sustainability in research and teaching programs. In approximately $70 \%$ of the cases, we could find 
evidence for institutionalization within the institution's structures, resulting in a mean value of 5.7 for this specific EMP dimension. The strongest mean value could be observed for the dimension environmental policy (6.3), followed by environmental processes (5.9) and environmental objectives (5.8). Interestingly, environmental monitoring appears to be an outlier, since the mean value of 5.1 lies noticeably below the other dimensions. Four of these seven institutions are also registered with EMAS. Differing from this pattern, two cases have high total EMP scores but no publicly available information on certain activities is provided. The mean value of high performers over all EMP dimensions is 5.8.

In the case of low-performing HEIs, the examination on the publicly available information delivered no further information on sustainability activities, neither in research and teaching, nor for the institutional context. Remarkably, almost two-thirds of the low performing HEIs are rather small, with only $10 \%$ of low-performing institutions being large. As was already observable within the high-performing HEIs, structures (2.2) show the highest adoption, followed by policy (2.0) and processes (1.9). Unlike the high-performer group, the mean monitoring performance (1.8) of low performers exceeds the performance on environmental objectives (1.7). Interestingly, we also found evidence for engagement in research or teaching or even both, which obviously did not result from an increased environmental management performance of the institution as such (cases 15 and 18). The mean value of low-performing institutions over all EMP dimensions is 1.6.

In the group of medium-performing HEIs, the results show a heterogeneous picture. Almost two-thirds of the medium-performers show an orientation towards sustainability, at least in research and teaching, in institutional bodies, or even both. The mean value for policy shows the highest value (4.4), followed by processes (4.4) and objectives (4.0). In contrast to the other performing groups, organizational structures (3.9) seem to be underdeveloped, while monitoring (3.6) appears to be relatively less common. Remarkably, in two cases, the study found no indication for actual engagement, which could explain the average results (cases 21 and 24). In contrast, the study also finds cases showing activities in research and teaching and in institutional anchoring (cases 1, 22 and 33). Surprisingly, none of the medium cases have undertaken EMAS registration. The mean value of medium performers is 4.0 , congruent with the median value of our scale.

\section{Discussion}

This survey study examines the incorporation and operationalization of environmental issues in HEIs' management and answers the research question of how HEIs conduct environmental management along the dimensions of EMP, including environmental policy, environmental objectives, environmental processes, organizational structures, and monitoring. The empirical data provide insights into the status quo of adoption and examine HEIs' environmental performance along the EMP dimensions of policy, objectives, structures, processes, and monitoring. The results show that environmental sustainability issues in fact play a role within HEIs' management, though the distribution across the different dimensions differs noticeably. The difference in performance levels between environmental objectives and environmental policy indicates a gap in the translation of (voluntary) commitments into clearly defined environmental objectives. The decoupling of these two aspects shows that although there is a basic awareness of the need to take environmental concerns into account, this awareness remains stuck on an abstract level and thus calls into question the actual will to commit oneself more, which ultimately leaves the impression of lip service. When looking at a whole-institutional approach, it becomes clear that although there is a willingness to assume responsibility at management level, this does not appear to be perceived as a core task of HEI action. In this understanding, efforts to establish supporting structures, processes or control mechanisms, in an effort to improve EMP, seem less promising in the long term.

Including the low average performance in terms of monitoring systems, a possible misfit with regard to effective management becomes noticeable (You cannot manage what you do not measure), which might be due to mutual reinforcement in conjunction with weak goal setting. 
Despite great efforts with a total of five rounds, only a relatively low response rate could be achieved among the HEIs surveyed. Although we exhausted all possibilities from a methodological point of view [28], we were not able to increase the response rate significantly. This may be due to the fact that there are no central contacts in the HEIs with regard to EMP. Since this survey was developed together with practical experts from the HE environmental management along the usual cascade of responsibilities for environmental issues in HEIs, the usual persons in charge seem only partially informed about the surveyed topics for the cross-section of HEIs. This leads to the conclusion that concrete contact persons, if any, are difficult to identify for the broad field of sustainability management. This may be due to a lack of consent on the managerial level, suggesting that holistic responsibility has not yet been regulated within the organization. This leads to the assumption that the spread of the cross-sectional function of sustainability management in HEIs has so far been low and a silo thinking (e.g., facility management, operations, focus on environmental or waste topics) according to defined responsibilities prevails.

With regard to the adoption of ecological sustainability in HEIs, another reason may be the lack of consent at the management level, which can be derived from a lack of relevance to the topic. There is some competitive pressure on HEIs with regard to integrating sustainability in research and teaching performance, but sustainability aspects, so far, are of minor relevance. Possible reasons could be that the public financing of HEIs through target agreements contains few or no sustainability targets. This means that the ministries do not tie any sustainability goals to the general budget of the public HEIs, thus the topic of sustainability plays a subordinate role. A similar result is reported in Heinicke and Guenther [31] for the implementation of management control systems. The authors argue that the fulfillment of political and legal requirements is of highest priority in order to secure funding for the HEI and it suppresses the adoption of management systems if not explicitly demanded or connected with funding.

Another reason for the relatively low and rather diverse adoption rates in our survey may be that sustainability management in HEIs is driven by the individual engagement of single players within the HEIs. This is confirmed by experiences of the authors in the research project HOCH-N. The HOCH-N project consists of a research network of 13 German universities as well as a number of committed institutions, organizations, and individuals from the academic environment who are dedicated to the question of sustainable development in the HE context. Respondents that are part of this network show a markedly sharper ecological sustainability profile in terms of policies, goals, structures, processes, and monitoring compared to HEIs that are not involved.

Our study contributes to the discussion of sustainability in HEIs in two ways. First, the study provides a survey-driven empirical approach for the systematization and relevance of environmental management aspects for determining the EMP of HEIs.

The vast majority of studies on environmental aspects of sustainability at HEIs, to date, have investigated success factors for the implementation of sustainability in the institution on a case-by-case basis. Due to the high contextual specifics, a generalization of the results has been possible only to a limited extent. The investigation of the introduction of sustainability from the ground up follows different development paths, mostly relating to the voluntary commitment of motivated actors. The present study allows to exceed the limitations of institutional specifics and to achieve a degree of generalizability. Furthermore, the study is oriented towards mechanisms of control as they are established in the corporate context as well as in new public management. Thus, the approach ties in with existing management structures and allows adopting a whole-institution approach from a management perspective. In addition to the examination of general success factors, the study shows where, from an organizational perspective, challenges for the implementation of sustainability can exist, and provides indications of how these can be mastered with the help of a performance-oriented approach.

The present study expands existing empirical research by investigating, by way of a broad survey, the occurrence of EMP in the context of HEIs. To the best of our knowledge, there are 
currently no other survey studies which have assessed the EMP of management bodies for the case of HEIs. Based on our full sample of HEIs from German-speaking countries, with no major concerns of response bias, the study expands the relevant literature by clarifying the importance of environmental management performance as a holistic (management) approach across the dimensions of EMP and its relevance to the measurement and management of environmental aspects at HEIs. It is evident that an organization-wide approach to managing environmental performance cannot be viewed solely at management or operational level, but must be implemented as a bipolar flow, flanked by voluntary engagement and stakeholder participation bottom-up and top-down in the organization if it is to be successful.

In view of the results of the study, it may also be useful to include aspects of organizational culture in the consideration. As Niedlich et al. [32] have shown in their study, organizational culture plays a crucial role in HEI's governance for the implementation of sustainability aspects. Cultural orientations at management level can thus act as a stimulus or barrier to the success or failure of implementation. With regard to EMP, it might be useful to consider these aspects of organizational culture.

In summary, the analysis shows an average moderate level of EMP and an interrelation between an increased EMP and ambitions for sustainable research and/or teaching.

Environmental policy shows, on average, the strongest emphasis, whereas monitoring systems tend to have received lesser attention. Furthermore, according to our results, the study discloses an association between the environmental performance level for EMP and an institution's size. Analyzing distinct fields of action and measures of environmental performance shows a moderate performance on disposal topics (mean value 4.49), energy efficiency (4.10), and facility management (4.04) issues. On the downside, the results show weak performance for marketing (2.96), HRM (3.05), and event management (3.25) topics.

A stronger performance in the field of waste disposal topics might be caused by the presence of distinct legal requirements regulating the waste management of (public) organizations and firms.

High performance levels in both functions of energy efficiency and facility management might result from a remarkable overlap in their practical execution. Since energy efficiency may also be included in an environmentally sustainable facility management, their mutual affection under the lens of practical operations appears to be evident. Another reason for these results might be due to low thresholds for taking action. In both cases, the implementation and operation of optimization potentials requires relatively short decision-making processes or top management commitment, but mainly personal engagement and willingness to contribute from the person(s) in charge of the function.

Remarkably, there is a missing entanglement with procurement issues (3.67) since maintenance requires appropriate supplies of operating resources. This actually contrasts with the performance results in the dimension of environmental processes (see Figure 6). Interestingly, more than half of the responding institutions have staff in charge of environmental (sustainability) issues (regardless of their size). According to our results, it seems that those functions are predominantly assigned to the HEI's facility office, since the study measured a strong EMP centered on central functions (especially facility management and disposal). However, this shows that an institutionalization of engagement does not lead automatically to an increase of the HEI's overall environmental management performance, since distinct fields of action and measures appear to perform weaker than others. This might also arise from a general lack of comprehensive systems of sustainability objectives, which require a holistic approach that includes all EMP dimensions.

As a second contribution, the study expands the current literature on sustainability in public HEIs by exploring the role of management for the implementation of (environmental) sustainability efforts. The application of EMP enables a new perspective for the understanding of aims and conditions of the successful implementation of a holistic sustainability approach within HEIs.

With a moderate adoption of environmental policies, processes and organizational structures, and a low dissemination of monitoring systems, EMP does not seem to have supported the change of perspective from an output orientation (e.g., reduction of waste and energy) to an outcome orientation 
for societal welfare (role model for students and researchers). With a general willingness to become active at the top management level, the operational practice (so far) is not sufficiently capable of fulfilling this commitment.

As the results show, environmental management is an emerging issue for HEIs in their endeavor to fulfill their social mission and reduce negative impacts of research and teaching. With the apparent willingness of management bodies to adapt environmental sustainability into their policies and structures, a first step towards the implementation of environmental management has already been made. However, the existing structures also must cover operational practices to pursue a holistic approach to implementing sustainability across the institution, thereby ensuring the lasting success of these efforts.

\section{Concluding Remarks}

Based on these findings, the study proposes distinct implications for both decision makers (e.g., administrators, environmental management officers, and the HEI's top management) and researchers. First, the pledge of engagement within the policy must be translated into certain goals guiding environmental officers and enabling the assessment of activities through monitoring systems, including the distribution of (financial) resources for the appropriate allocation of means to implement sustainability. Importantly, measuring the allocation and use of means is crucial for building long-term structures enabling continuous (improvement) processes. Applying monitoring systems in that context not only contributes to assessing the EMP, but also enhances transparency and broadcasts information on the progress of implementation and improvement, improving the social credibility needed to pursue the intended policies and objectives.

Second, the results suggest the charge of environmental sustainability within HEIs is complex. It seems that the success of certain efforts depends on the setting of the person(s) in charge of these issues. Apparently, environmental sustainability seems to be located within the facility division in most cases. This may be a logical consequence of the importance of this field of action, which, however, likewise neglects other fields. A possible solution might be the implementation of cross-sectional posts that can manage and control all relevant fields of action and measures of environmental sustainability simultaneously. Another option describes participatory approaches supporting existing structures to implement policies and objectives by voluntary engagement or shared governance. Since distinct stakeholder groups, such as students or staff members, are directly affected by HEIs and therefore have a legitimate claim to participate, their involvement on a voluntary basis could have the potential to foster environmental engagement and integrate relevant target groups into decision-making processes.

The aforementioned points to the third implication on continuity and impact. Since our study observes a weak anchoring of environmental objectives and an even weaker embedding of monitoring and control mechanisms, the measurement and assessment performance of environmental issues under the lens of EMP remains limited to short or mid-term effects. Generating impacts should actually be the standard for engagement, but appropriate indicators or measurement tools are still missing. Nevertheless, the fact that HEIs have established structures suggests a positive development and shows their willingness to contribute and shoulder responsibility. Turning to impact measurement, the question arises as to how HEIs can pursue purpose through their actions.

As many other surveys, our study also has limitations providing avenues for future research. One major limitation in the generalizability of the results lays in the fact that only German-speaking and state-run HEIs are taken into account. Public sponsorship and state funding are a decisive differentiating factor in comparison with other countries and also in contrast to private HEIs. Future research may assess whether our findings also hold for other regions and HEI settings. Another major limitation is the relatively low total number of responses which does not allow for statistical testing of our findings. Thus, interpretation of results has to be conducted carefully. In addition, the unit response tests indicate that the sample contains more universities than universities of applied science than expected, which could bias our findings. Therefore, future research could examine the EMP for other 
countries or HEI cultures (e.g., UK or US). Furthermore, future researchers could draw a comparison between private and public institutions. A replication of our HE setting in other global regions, as well as a comparison with other educational systems (e.g., North America, Australia, and Asia) could also be an interesting endeavor. It is also conceivable to expand our survey to performance issues in teaching and research in relation to the operational performance of an HEI to assess the overall sustainability performance of an HEI.

Another future task will be to define an HEI's responsibilities to planet and people beyond the core business of teaching and research. This opens the field of whether the terms sustainability and responsibility can be regarded as complementary or as competing terms associated with different functions. Thus, a future research question could cover whether it is useful to introduce an overarching sustainability (performance) management on all dimensions of the triple bottom line or if treating each dimension (i.e., environmental and social sustainability) separately is more appropriate. Based on the results, HEIs are moving in the right direction, even though there is a considerable potential for institutions to improve further, and ensure this progress continues long into the future.

Author Contributions: Conceptualization, N.R., X.H., E.G., and T.W.G.; methodology, X.H. and N.R.; software, X.H. and N.R.; validation, X.H. and N.R.; formal analysis, X.H. and N.R.; resources, E.G. and T.W.G.; data curation, N.R. and X.H.; writing—original draft preparation, N.R. and X.H.; writing—review and editing, N.R., X.H., E.G., and T.W.G.; visualization, X.H. and N.R.; supervision, E.G. and T.W.G.; project administration, N.R. All authors have read and agree to the published version of the manuscript.

Funding: This research received no external funding.

Acknowledgments: We thank the editor and reviewers for their valuable and helpful feedback on this publication.

Conflicts of Interest: The authors declare no conflict of interest.

\section{References}

1. Amaral, L.P.; Martins, N.; Gouveia, J.B. Quest for a sustainable university: A review. Int. J. Sustain. High. Educ. 2015, 16, 155-172. [CrossRef]

2. Figueiró, P.S.; Raufflet, E. Sustainability in higher education: A systematic review with focus on management education. J. Clean. Prod. 2015, 106, 22-33. [CrossRef]

3. Alshuwaikhat, H.M.; Abubakar, I. An integrated approach to achieving campus sustainability: Assessment of the current campus environmental management practices. J. Clean. Prod. 2008, 16, 1777-1785. [CrossRef]

4. Disterheft, A.; da Silva, S.S.F.; Ramos, M.R.; Azeiteiro, U.M.d. Environmental Management Systems (EMS) implementation processes and practices in European higher education institutions-Top-down versus participatory approaches. J. Clean. Prod. 2012, 31, 80-90. [CrossRef]

5. Sammalisto, K.; Sundström, A.; Holm, T. Implementation of sustainability in universities as perceived by faculty and staff - A model from a Swedish university. J. Clean. Prod. 2015, 106, 45-54. [CrossRef]

6. Brinkhurst, M.; Rose, P.; Maurice, G.; Ackerman, J.D. Achieving campus sustainability: Top-down, bottom-up, or neither? Int. J. Sustain. High. Educ. 2011, 12, 338-354. [CrossRef]

7. Roos, N.; Guenther, E. Sustainability Management Control Systems in Higher Education Institutions. From Measurement to Management. IJSHE 2020, 21. [CrossRef]

8. Trumpp, C.; Endrikat, J.; Zopf, C.; Guenther, E. Definition, Conceptualization, and Measurement of Corporate Environmental Performance: A Critical Examination of a Multidimensional Construct. J. Bus. Ethics 2015, 126, 185-204. [CrossRef]

9. Clarke, A.; Kouri, R. Choosing an appropriate university or college environmental management system. J. Clean. Prod. 2009, 17, 971-984. [CrossRef]

10. Leal Filho, W.; Wu, Y.C.J.; Brandli, L.L.; Avila, L.V.; Azeiteiro, U.M.; Caeiro, S.; Madruga, L.R.D.R.G. Identifying and overcoming obstacles to the implementation of sustainable development at universities. J. Integr. Environ. Sci. 2017, 14, 93-108. [CrossRef]

11. Lozano, R. Incorporation and institutionalization of $S D$ into universities: Breaking through barriers to change. J. Clean. Prod. 2006, 14, 787-796. [CrossRef]

12. Savely, S.M.; Carson, A.I.; Delclos, G.L. An environmental management system implementation model for U.S. colleges and universities. J. Clean. Prod. 2007, 15, 660-670. [CrossRef] 
13. Berzosa, A.; Bernaldo, M.O.; Fernández-Sanchez, G. Sustainability assessment tools for higher education: An empirical comparative analysis. J. Clean. Prod. 2017, 161, 812-820. [CrossRef]

14. Lozano, R. A tool for a Graphical Assessment of Sustainability in Universities (GASU). J. Clean. Prod. 2006, 14, 963-972. [CrossRef]

15. Burrell, D.N.; Anderson, M.; Bessette, D.; Dawson, M. A Contemporary Evaluation of Universities and Sustainability Strategic Planning. Rev. Manag. Innov. Creat. 2011, 4, 65-80.

16. Speklé, R.F.; Widener, S.K. Challenging Issues in Survey Research: Discussion and Suggestions. J. Manag. Account. Res. 2018, 30,3-21. [CrossRef]

17. Posner, S.M.; Stuart, R. Understanding and advancing campus sustainability using a systems framework. Int. J. Sustain. High. Educ. 2013, 14, 264-277. [CrossRef]

18. Ribeiro, M.M.; Hoover, E.; Burford, G.; Buchebner, J.; Lindenthal, T. Values as a bridge between sustainability and institutional assessment: A case study from BOKU University. Int. J. Sustain. High. Educ. 2015, 17, 40-53. [CrossRef]

19. Kehm, B.M.; Lanzendorf, U. The impacts of university management on academic work: Reform experiences in Austria and Germany. Manag. Rev. 2007, 18, 153-173. [CrossRef]

20. Dillman, D.A.; Smyth, J.D.; Christian, L.M. Internet, phone, mail and mixed-mode surveys: The tailored design method. Reis 2016, 154, 161-176.

21. Brislin, R.W. Back-translation for cross-cultural research. J. Cross-Cult. Psychol. 1970, 1, 185-216. [CrossRef]

22. Guenther, T.W.; Heinicke, A. Relationships among types of use, levels of sophistication, and organizational outcomes of performance measurement systems: The crucial role of design choices. Manag. Account. Res. 2019, 42, 1-25. [CrossRef]

23. Van der Stede, W.A.; Young, S.M.; Chen, C.X. Assessing the quality of evidence in empirical management accounting research: The case of survey studies. Account. Organ. Soc. 2005, 30, 655-684. [CrossRef]

24. Armstrong, J.S.; Overton, T.S. Estimating nonresponse bias in mail surveys. J. Mark. Res. 1977, 14, $396-402$. [CrossRef]

25. Bisbe, J.; Malagueño, R. Using strategic performance measurement systems for strategy formulation: Does it work in dynamic environments? Manag. Account. Res. 2012, 23, 296-311. [CrossRef]

26. De Geuser, F.; Mooraj, S.; Oyon, D. Does the balanced scorecard add value? Empirical evidence on its effect on performance. Eur. Account. Rev. 2009, 18, 93-122. [CrossRef]

27. Libby, T.; Lindsay, R.M. Beyond budgeting or budgeting reconsidered? A survey of North-American budgeting practice. Manag. Account. Res. 2010, 21, 56-75. [CrossRef]

28. Hiebl, M.R.W.; Richter, J.F. Response Rates in Management Accounting Survey Research. J. Manag. Account. Res. 2018, 30, 59-79. [CrossRef]

29. Van der Stede, W.A.; Young, S.M.; Chen, C.X. Doing management accounting survey research. Handb. Manag. Account. Res. 2006, 1, 445-478.

30. Henri, J. Taxonomy of performance measurement systems. In Advances in Management Accounting; Elsevier: Amsterdam, The Netherlands, 2009; Volume 17, pp. 247-288.

31. Heinicke, X.; Guenther, T.W. The Role of Management Controls in the Higher Education Sector: An Investigation of Different Perceptions. Eur. Account. Rev. 2019, 1-50. [CrossRef]

32. Niedlich, S.; Kummer, B.; Bauer, M.; Rieckmann, M.; Bormann, I. Cultures of sustainability governance in higher education institutions: A multi-case study of dimensions and implications. High. Educ. Q. 2019. [CrossRef]

(C) 2020 by the authors. Licensee MDPI, Basel, Switzerland. This article is an open access article distributed under the terms and conditions of the Creative Commons Attribution (CC BY) license (http://creativecommons.org/licenses/by/4.0/). 